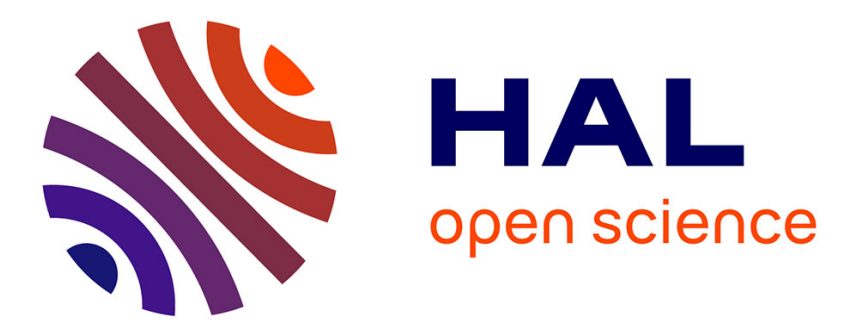

\title{
Dynamically transitioning between surfaces of varying inclinations to achieve uneven-terrain walking
} Luca Colasanto, Nicolas Perrin, Nikos G Tsagarakis, Darwin G Caldwell

\section{To cite this version:}

Luca Colasanto, Nicolas Perrin, Nikos G Tsagarakis, Darwin G Caldwell. Dynamically transitioning between surfaces of varying inclinations to achieve uneven-terrain walking. 2014 IEEE International Conference on Robotics and Automation (ICRA), May 2014, Hong Kong, China. pp.210 - 215, 10.1109/ICRA.2014.6906611 . hal-01394896

\section{HAL Id: hal-01394896 https://hal.sorbonne-universite.fr/hal-01394896}

Submitted on 10 Nov 2016

HAL is a multi-disciplinary open access archive for the deposit and dissemination of scientific research documents, whether they are published or not. The documents may come from teaching and research institutions in France or abroad, or from public or private research centers.
L'archive ouverte pluridisciplinaire HAL, est destinée au dépôt et à la diffusion de documents scientifiques de niveau recherche, publiés ou non, émanant des établissements d'enseignement et de recherche français ou étrangers, des laboratoires publics ou privés. 


\title{
Dynamically transitioning between surfaces of varying inclinations to achieve uneven-terrain walking*
}

\author{
Luca Colasanto $^{1,2}$, Nicolas Perrin ${ }^{3}$, Nikos G. Tsagarakis ${ }^{1}$ and Darwin G. Caldwell ${ }^{1}$
}

\begin{abstract}
This paper focuses on how to generate dynamic transitions in order to make our robot COMAN (COmpliant huMANoid) dynamically traverse inclined terrains. The novel approach addresses dynamic walking on inclined surfaces by dividing the walking motion into two phases: transition and incline walking. During the transition phase, the humanoid robot performs a 3-dimensional movement in order to transfer its body between surfaces of different inclinations, which is then followed by the incline-walking phase. The transition phase is less trivial to execute than the incline walking itself. In this paper, we first formulate the equations of a 3D (non linear) Inverted Pendulum, and then we derive an equivalent model. Following this we introduce a trajectory generator based on this model and validate it experimentally by performing, with COMAN, dynamic transitions from the horizontal ground to a $10^{\circ}$ slope.
\end{abstract}

\section{INTRODUCTION}

Humanoid locomotion has been extensively studied over the past forty years, and there exist now various approaches to perform dynamic walking on flat ground. However, the state-of-the-art techniques used on flat terrains cannot be directly applied to achieve walking on uneven terrains, which is still a challenging problem for researchers. Attempts to solve this problem generally treat separately the two following phases: the "transition phase" and the "incline walking" phase. During the transition phase, the robot performs a 3dimensional motion in order to transfer its body between surfaces of different inclinations, and after that, the inclinewalking phase starts. Traditional models used for flat ground walking (generally involving Zero Moment Point [1] and Linear Inverted Pendulum [2]) can usually be generalized to deal with incline walking itself, nevertheless the transition phase is more complicated to handle, for several reasons. First, as most humanoids are unable to perform heel toe strikes, transition phases often requires a large range of motion for the ankle, knee and hip joints of the robot. Besides, large torques are usually necessary, and finally linear models are less suitable to represent the motions that must be performed. In fact, traditional linear models simplify the dynamics by constraining the vertical movement to have zero acceleration. A first solution commonly adopted consists in performing the transition movement mostly in double support (when both feet are on the ground and the support polygon is larger), and using quasi-static movements

\footnotetext{
*This work is supported by the FP7 European project Walk-Man (FP7ICT-611832).

${ }^{1}$ Department of Advanced Robotics, Istituto Italiano di Tecnologia, via Morego, 30, 16163 Genova.

${ }^{2}$ Biorobotics Laboratory, Institute of Bioengineering, School of Engineering, Ecole Polytechnique Fédérale de Lausanne (EPFL), Switzerland.

${ }^{3}$ Institut des Systèmes Intelligents et de Robotique, CNRS UMR 7222 \& Université Pierre et Marie Curie, 4 place Jussieu, 75252 Paris cedex 05, France.
}

in single support [3]. In this way, the robot needs to stop or reduce considerably its speed at proximity of the edge, but provided that the robot can produce enough torque, this approach can usually deal with larger inclination changes than other more dynamic existing methods. Lim et al. [4] and Seven et al. [5] address transition phases as pure control problems, considering slopes as disturbances. With their approaches, they manage to cope with inclination changes of $7^{\circ}$ and $8.5^{\circ}$.

In our novel approach, we exploit the natural dynamics of a 3D (non linear) Inverted Pendulum and we develop an "equivalent" model. This model is used to design trajectories that capture well the dynamics of the system while respecting constraints fixed by a desired gait. Performing the transition movements mostly during the single support phase and including the effect of the vertical movement of the center of mass in the dynamics of the system, we were able to make the robot traverse inclined terrains without changing the duration of the gait cycle.

The lower body of the new COmpliant huMANoid robot, COMAN, was used to perform the tests. Its intrinsic compliance, due to the passive elasticity in its joints, makes this robot a good candidate for this kind of task. In previous works [6], [7], [8] we focused on the effects of compliance on the dynamics of walking. It was demonstrated that compliance can reduce the effect of disturbances due to the take off and landing of the foot. In fact, instead of a stiff response to the disturbance it introduces a proportional action to the position error (as an intrinsic proportional controller).

In the following sections, we first derive the equations of a 3D Inverted Pendulum, and the "equivalent model". After, a trajectory generator is developed. Following that, the experimental setup is explained and the results of walking, showing dynamic transition on a $10^{\circ}$ inclined surface, are reported and discussed.

\section{THE 3D INVERTED PENDULUM MODEL}

In this section the equations of the 3D (non linear) inverted pendulum model, which is used to approximate the robot in this work, are described and then an equivalent representation is derived.

\section{A. Model Equations}

The problem of an inverted pendulum anchored to a surface generally oriented in the world and subject to gravity is described in the following equation:

$$
\tau_{g r}+r_{C O M} \times G=\frac{\mathrm{d}}{\mathrm{d} t}\left(r_{C O M} \times L\right)
$$

where $\tau_{g r} \in \mathfrak{R}^{3}$ represent the torques at the contact point between the ground and the inverted pendulum, $r_{C O M} \in \mathfrak{R}^{3}$ 


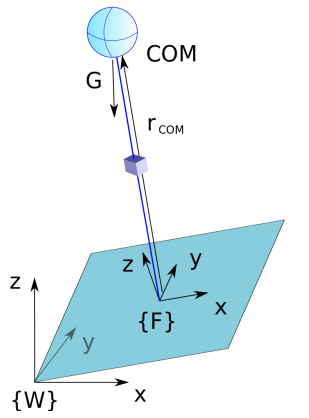

Fig. 1. 3D Inverted Pendulum Model.

is the position of the COM, $G \in \mathfrak{R}^{3}$ is the gravitational force and $\mathrm{L}$ is the linear momentum. These variables are expressed with respect to a Cartesian reference frame $\{\mathrm{F}\}$ that has its origin at the contact point with the $\mathrm{x}$ and $\mathrm{y}$ axes parallel to the surface. Referring to equation (1) the inverted pendulum model is depicted in Fig. 1.

We define $\alpha_{1}, \alpha_{2}$ and $\alpha_{3}$ as respectively the roll, pitch and yaw angles of the inclined surface, for example if the surface is inclined upward, $\alpha_{1}$ will be positive. Then the gravitational force, expressed in $\{\mathrm{F}\}$ can be represented as follows:

$$
G=m\left[\begin{array}{c}
-g c_{1} c_{3}-g s_{2} s_{3} \\
g s_{1} s_{3}-g s_{2} c_{3} \\
-g c_{1} c_{2}
\end{array}\right]=m\left[\begin{array}{l}
g_{x} \\
g_{y} \\
g_{z}
\end{array}\right]
$$

where $\mathrm{m}$ is the mass of the inverted pendulum and $c_{1}, c_{2}$, $c_{3}, s_{1}, s_{2}, s_{3}$ are respectively the cosine and sine functions of the angles $\alpha_{1}, \alpha_{2}$ and $\alpha_{3}$.

The definition of Zero Moment Point (ZMP) can be then formulated as follows:

$$
\tau_{g r}-r_{z m p} \times G=\left[\begin{array}{c}
0 \\
0 \\
M_{z}
\end{array}\right]
$$

where $r_{z m p}=\left[\begin{array}{lll}x_{z m p} & y_{z m p} & 0\end{array}\right]^{T}$ is the ZMP position in $\{\mathrm{F}\}$, and $M_{z}$ is the vertical momentum.

Solving the equation (2) with respect to $\tau_{g r}$ and substituting it into equation (1) the dynamic system of the inverted pendulum in Fig. 1 can be expressed as follows:

$$
\left\{\begin{array}{l}
\ddot{x} z=\left(\ddot{z}-g_{z}\right) x+g_{x} z+g_{z} x_{z m p} \\
\ddot{y} z=\left(\ddot{z}-g_{z}\right) y+g_{y} z+g_{z} y_{z m p}
\end{array}\right.
$$

\section{B. Equivalent Representation}

The first differential equation of (3) represents the movement of the COM along the $\mathrm{x}$ direction of the reference frame $\{\mathrm{F}\}$. It can be written using the state space representation as follows:

$$
\begin{aligned}
{\left[\begin{array}{c}
\dot{x} \\
\ddot{x}
\end{array}\right] } & =\left[\begin{array}{cc}
0 & 1 \\
\frac{\ddot{z}-g_{z}}{z} & 0
\end{array}\right]\left[\begin{array}{l}
x \\
\dot{x}
\end{array}\right]+\left[\begin{array}{c}
0 \\
g_{x}+\frac{g_{z}}{z} x_{z m p}
\end{array}\right] \\
& =\mathbf{A}(t) X(t)+B(t)
\end{aligned}
$$

It is a second order nonlinear dynamic system with parameters: $\alpha_{1}, \alpha_{2}, \alpha_{3}, z, \dot{z}, \ddot{z}$ and $x_{z m p}$. In particular, the first three parameters are constant while the others are time variants. In order to have a diagonal state-transition matrix (it simplifies the computation of the solution) we define a state transformation:

$$
\begin{array}{r}
\hat{X}=\mathbf{T}^{-1} X=\left[\begin{array}{cc}
\frac{1}{2} & \frac{1}{2} \\
\frac{1}{2 f_{2}} & -\frac{1}{2 f_{2}}
\end{array}\right]^{-1} X \\
\hat{\mathbf{A}}=\mathbf{T}^{-1} \mathbf{A} \mathbf{T}=\left[\begin{array}{cc}
f_{1} & 0 \\
0 & -f_{1}
\end{array}\right] \\
\hat{B}=\mathbf{T}^{-1} B=\left[\begin{array}{c}
f_{2}\left(g_{x}+\frac{g_{z}}{z} x_{z m p}\right) \\
-f_{2}\left(g_{x}+\frac{g_{z}}{z} x_{z m p}\right)
\end{array}\right]
\end{array}
$$

where:

$$
f_{1}=\frac{\sqrt{z\left(\ddot{z}-g_{z}\right)}}{z} \quad f_{2}=\frac{\sqrt{z\left(\ddot{z}-g_{z}\right)}}{\ddot{z}-g_{z}}
$$

Since $z\left(\ddot{z}-g_{z}\right)>0$, the invertibility of the transformation matrix $\mathbf{T}$ (and hence the uniqueness of the transformation) is guaranteed. This condition is valid in a wide range of applications. In fact, $z$ is positive in all the feasible configuration of the inverted pendulum. Moreover, in the case of $\ddot{z} \geq 0$, the condition is valid in the range of parameters:

$$
-\frac{\pi}{2} \leq \alpha_{1} \leq \frac{\pi}{2} \quad-\frac{\pi}{2} \leq \alpha_{2} \leq \frac{\pi}{2}
$$

All the configurations that can be exploited with the real robot do not violate these constraints. Instead, in the case of $\ddot{z}<0$, the condition results valid if the following constraint is verified:

$$
\frac{|\ddot{z}|}{g}<c_{1} c_{2}
$$

This constraint is generally satisfied because the ratio, between the COM accelerations and the constant $g$, has low values during walking. For instance, the maximum acceleration, during a walk performed by a human being, is around $2 \mathrm{~m} / \mathrm{s}^{2}$ (in the direction of walking), thus the ratio is less than 0.2 . Therefore, the condition for the invertibility of the transformation matrix $\mathbf{T}$ is assumed always true. The equivalent system can be represented as follows:

$$
\dot{\hat{X}}=\hat{\mathbf{A}} \hat{X}+\hat{B}
$$

The solution of (6) is given by the following equations:

$$
\begin{aligned}
& \hat{x}_{1}(t)=e^{\int_{0}^{t} f_{1}(u) d u} \hat{x}_{1}(0) \\
& \quad+\int_{0}^{t} e^{\int_{v}^{t} f_{1}(u) d u} f_{2}(v)\left(g_{x}+\frac{g_{z}}{z(v)} x_{z m p}(v)\right) d v \\
& \hat{x}_{2}(t)=e^{-\int_{0}^{t} f_{1}(u) d u} \hat{x}_{2}(0) \\
& \quad-\int_{0}^{t} e^{-\int_{v}^{t} f_{1}(u) d u} f_{2}(v)\left(g_{x}+\frac{g_{z}}{z(v)} x_{z m p}(v)\right) d v
\end{aligned}
$$

where $\hat{x}_{1}(t)$ and $\hat{x}_{2}(t)$ are respectively the first and the second elements of the equivalent state vector $\hat{X}$.

A similar procedure was applied to the second differential equation of (3). It represents the movement of the COM along the y direction (Fig. 1). The solution of the equivalent system is given by:

$$
\begin{aligned}
& \hat{y}_{1}(t)=e^{\int_{0}^{t} f_{1}(u) d u} \hat{y}_{1}(0) \\
& \quad+\int_{0}^{t} e^{\int_{v}^{t} f_{1}(u) d u} f_{2}(v)\left(g_{y}+\frac{g_{z}}{z(v)} y_{z m p}(v)\right) d v \\
& \hat{y}_{2}(t)=e^{-\int_{0}^{t} f_{1}(u) d u} \hat{y}_{2}(0) \\
& \quad-\int_{0}^{t} e^{-\int_{v}^{t} f_{1}(u) d u} f_{2}(v)\left(g_{y}+\frac{g_{z}}{z(v)} y_{z m p}(v)\right) d v
\end{aligned}
$$




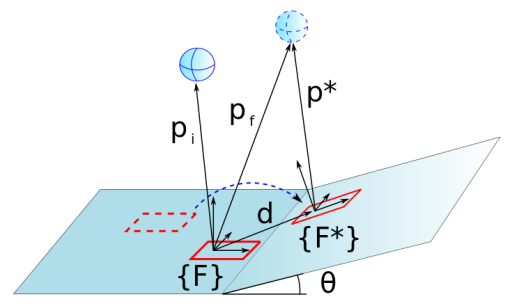

Fig. 2. Schematics of the transition phase.

The equivalence used for the y movement is the same used for the $\mathrm{x}$ movement.

\section{TRAJECTORY GENERATOR}

We approximate the robot using the 3D inverted pendulum as in Fig. 1. The $\{F\}$ is located at the foot of the supporting leg and the mass at the COM of the robot. In this work we focus on the case where the inclination changes in just one direction. In the following, we refer to the common case where the robot is walking on a flat surface and then a positive slope (with inclination equal to $\theta$ ) occurs. However the method presented is general and can be used in any situation.

The transition movement (of duration $T_{t r}$ ) starts with a single support phase (of duration $T_{s s}$ ) where the robot brings one foot on the slope. Then follows the double support phase (of duration $T_{d b}$ ) where the robot has one foot on the flat ground and another on the slope. The trajectories to perform during the first phase are defined by equations (7) and (8) while the trajectories used for the second phase are polynomial trajectories.

First, the initial and final position and velocity of the COM of the robot during the transition movement are computed, then the vertical movement (along $\mathrm{z}$ direction) is defined. Finally, the ZMP trajectories to drive the movement during the single support are computed using the equivalent representation of the Inverted Pendulum.

\section{A. Initial and Final Position and Velocity}

We generate the trajectories for walking on the flat ground or on the inclined surface using the 3D Linear Inverted Pendulum model [2] and the ZMP criterion to guarantee the stability.

Referring to Fig. 2, $\{F\}$ is located at the support foot of the transition movement and $p_{i}=\left[x_{i}, y_{i}, z_{i}\right]^{T}$ is the initial position of the transition movement. $d$ is the position (w.r.t. $\{F\}$ ) where the second foot is supposed to land at the end of the single support phase and $\left\{F^{*}\right\}$ is the reference frame of the support foot of the first walking cycle on the incline surface. $p^{*}$ is the position of the COM (w.r.t. $\left\{F^{*}\right\}$ ) at the beginning of the walking on the incline surface. The final position of the transition movement can be computed as $p_{f}=d+R_{y}(-\theta) p^{*}$ where $R_{y}(\cdot)$ is the rotational matrix around the y axis. In the same way the final velocity of the transition movement can be computed as $\dot{p}_{f}=\left[\dot{x}_{f}, \dot{y}_{f}, \dot{z}_{f}\right]^{T}=R_{y}(-\theta) \dot{p}^{*}$ where $\dot{p}^{*}$ is the velocity of the COM (w.r.t. $\left\{F^{*}\right\}$ ) at the beginning of the walking on the incline surface.

\section{B. Vertical Movement and ZMP Trajectories}

Given the desired duration of the entire transfer phase $\left(T_{t r}\right)$ we define the trajectory for the vertical movement of the

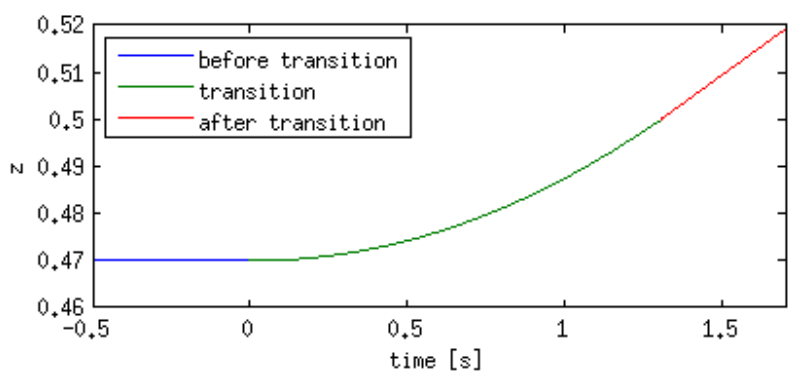

Fig. 3. $\mathrm{z}$ trajectory : $T_{t r}=1.3 \mathrm{~s}, z(0)=0.470 \mathrm{~m}, \dot{z}(0)=0, z\left(T_{t r}\right)=0.500 \mathrm{~m}$, $\dot{z}\left(T_{t r}\right)=0.039 \mathrm{~s}$

COM as follows:

$$
\begin{aligned}
z(t)= & a_{1} t^{2}+a_{2} t+a_{3} \quad t=\left[0: T_{t r}\right] \\
a_{3} & =z(0) \\
a_{2} & =\frac{\dot{z}(0) T_{t r}+2 z\left(T_{t r}\right)-2 a_{3}+\dot{z}\left(T_{t r}\right) T_{t r}}{2 T_{t r}} \\
a_{1} & =\frac{z\left(T_{t r}\right)-a_{2} T_{t r}-a_{3}}{T_{t r}^{2}}
\end{aligned}
$$

This is a second order polynomial trajectory that best fits the desired constraints $\left(T_{t r}, z(0), z\left(T_{t r}\right), \dot{z}(0)\right.$ and $\left.\dot{z}\left(T_{t r}\right)\right)$. The function to use for the vertical movement can also be different from (9). However the proposed one ensures the invertibility of the transformation matrix $\mathbf{T}$ (see II-B), and it allows some later simplification in the computation because of its low order (see III-C).

Fig. 3 reports an example of trajectory generated with (9). Negative time represents instants before the transition (blue), then the trajectory during the transition is reported in green and after the transition in red.

The ZMP trajectories are defined as follows:

$$
\begin{aligned}
x_{z m p}(t)= & \beta \\
y_{z m p}(t)= & \begin{cases}\gamma \quad \text { if } & 0 \leq t \leq T_{s s} \\
-\gamma \text { if } & T_{s w}<t \leq T_{s w}\end{cases} \\
& T_{s w} \leq T_{s s}<T_{t r}
\end{aligned}
$$

where $\beta, \gamma, T_{s w}$ are parameters which concern the ZMP trajectory while $T_{s s}$ refers to the duration of the single support. The ZMP reference for the $\mathrm{x}$ direction is a constant value while for the $\mathrm{y}$ direction, step functions are used.

\section{Parameters Computation}

In order to compute the trajectory of the movement along the $\mathrm{x}$ and $\mathrm{y}$ directions the values of the parameters $\beta, \gamma, T_{s w}$ and $T_{s s}$ need to be computed.

The values of the parameters $\beta$ and $T_{s s}$ are computed considering the equations of the movement along $\mathrm{x}$. We define a candidate function for the double support phase as follows

$$
\begin{gathered}
x_{d b}(t)=\left(b_{1}\left|t-\frac{T_{t r}}{2}\right|+b_{2}\right)\left(t-\frac{T_{t r}}{2}\right) \quad t=\left[0: T_{t r}\right] \\
b_{1}=\frac{2 \dot{x}_{f} T_{t r}-4 x_{f}}{T_{t r}^{2}} \\
b_{2}=\dot{x}_{f}-b_{1} T_{t r}
\end{gathered}
$$




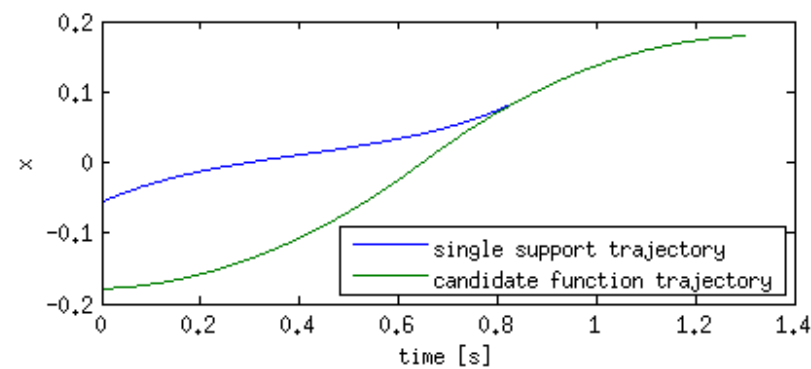

Fig. 4. $\mathrm{x}$ trajectory and candidate function : $x(0)=-0.057 m, \dot{x}(0)=0.320$, $x\left(T_{t r}\right)=0.189 \mathrm{~m}, \dot{x}\left(T_{t r}\right)=0.146, T_{t r}=1.3 \mathrm{~s}, T_{s s}=0.93 \mathrm{~s}, \beta=0.105 \mathrm{~m}$

This function of class $C^{1}$ is symmetrical with respect to the point $\left(\frac{T_{t r}}{2}, 0\right)$, and takes the value $x_{f}$ with derivative $\dot{x}_{f}$ at $t=T_{t r}$. We refer to it as a "candidate function" because just part of it is used during the double support phase, more specifically the part from the computed value $t=T_{s s}$ to $t=$ $T_{t r}$.

Substituting (10) into (7), considering (12) and (5), and after some manipulation of the equations, the following system is derived:

$$
\left\{\begin{array}{c}
\beta=\frac{\frac{\hat{x}_{d b}\left(T_{s s}\right)}{e^{F_{1}\left(T_{s s}\right)}}-e^{-F_{1}(0)} \hat{x}_{1}(0)-g_{x} \int_{0}^{T_{s s}} e^{-F_{1}(v)} f_{2}(v) d v}{g_{z} \int_{0}^{T_{s s}} e^{-F_{1}(v)} \frac{f_{2}(v)}{z(v)} d v} \\
\beta=\frac{\frac{\hat{\dot{x}}_{d b}\left(T_{s s}\right)}{e^{-F_{1}\left(T_{s s}\right)}}-e^{F_{1}(0)} \hat{x}_{2}(0)+g_{x} \int_{0}^{T_{s s}} e^{F_{1}(v)} f_{2}(v) d v}{-g_{z} \int_{0}^{T_{s s}} e^{F_{1}(v)} \frac{f_{2}(v)}{z(v)} d v}
\end{array}\right.
$$

were $F_{1}(\cdot)$ is the primitive of $f_{1}(\cdot)$ and $\hat{x}_{d b}(t)$ and $\hat{\dot{x}}_{d b}(t)$ are obtained applying the transformation (5) respectively to $x_{d b}(t)$ and $\dot{x}_{d b}(t)$. Because of we kept the order of $z(t)$ lower than three (see III-B), the primitive of $f_{1}(t)$ exists and is defined as follows:

$$
\begin{array}{r}
F_{1}(t)=\sqrt{\frac{2 a_{1}-g_{z}}{a_{1}}} \ln \left(\sqrt{\left(2 a_{1}-g_{z}\right)\left(a_{1} t^{2}+a_{2} t+a_{3}\right)}\right. \\
\left.+\frac{\left(2 a_{1} t+a_{2}\right)\left(2 a_{1}-g_{z}\right)}{2 \sqrt{a_{1}\left(2 a_{1}-g_{z}\right)}}\right)
\end{array}
$$

Therefore, (13) is a system of two equation in two unknown, $T_{s s}$ and $\beta$ respectively. More specifically, this system allows to compute the time $T_{s s}<T_{t r}$ where the single support trajectory can "join" the double support candidate function together with the corresponding value of $\beta$. Then, the trajectory of the whole movement along the $\mathrm{x}$ direction can be expressed as follows:

$$
x_{t r}(t)= \begin{cases}x(t) & \text { if } 0 \leq t \leq T_{s s} \\ x_{d b}(t) & \text { if } T_{s s}<t \leq T_{t r}\end{cases}
$$

Fig. 4 reports an example of our candidate function (in green) for the movement along the $\mathrm{x}$ direction and the trajectory developed for the single support (in blue). These results are computed based on the $\mathrm{z}$ trajectory reported in Fig. 3.

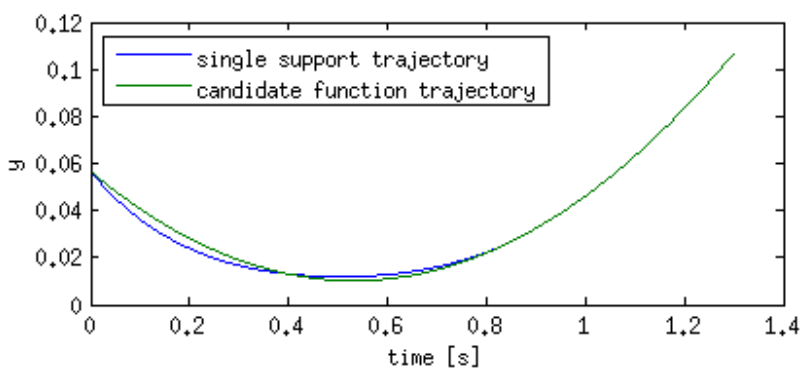

Fig. 5. y trajectory and candidate function : $y(0)=0.057 m, \dot{y}(0)=-0.253$, $y\left(T_{t r}\right)=0.107 \mathrm{~m}, \dot{y}\left(T_{t r}\right)=0.251, T_{s s}=0.96 \mathrm{~s}, T_{s w}=0.340 \mathrm{~s}, \gamma=0.001 \mathrm{~m}$

In a similar way the setting of the parameters $\gamma$ and $T_{s w}$ is addressed using the equations of the movement along $\mathrm{y}$. The candidate function for the double support is defined as follows

$$
\begin{gathered}
y_{d b}(t)=b_{1} t^{2}+b_{2} t+b_{3} \quad t=\left[0: T_{t r}\right] \\
b_{1}=\frac{\dot{y}_{f} T_{t r}+b_{3}-y_{f}}{T_{t r}^{2}} \\
b_{2}=\dot{y}_{f}-2 b_{1} T_{t r} \\
b_{3}=y_{i}
\end{gathered}
$$

It is a second order polynomial function (class $C^{\infty}$ ) taking the value $y_{f}$ and derivative $\dot{y}_{f}$ at $t=T_{t r}$.

Substituting (11) into (8), referring to (16) and (5), the following system is derived:

$$
\left\{\begin{array}{c}
\gamma=\frac{\frac{\hat{y}_{d b}\left(T_{s s}\right)}{e^{F_{1}\left(T_{s s}\right)}}-e^{-F_{1}(0)} \hat{y}_{1}(0)-g_{y} \int_{0}^{T_{s s}} e^{-F_{1}(v)} f_{2}(v) d v}{2 g_{z} \int_{0}^{T_{s w}} e^{-F_{1}(v)} \frac{f_{2}(v)}{z(v)} d v-g_{z} \int_{0}^{T_{s s}} e^{-F_{1}(v)} \frac{f_{2}(v)}{z(v)} d v} \\
\gamma=\frac{\frac{\hat{\dot{y}}_{d b}\left(T_{s s}\right)}{e^{-F_{1}\left(T_{s s}\right)}}-e^{F_{1}(0)} \hat{y}_{2}(0)+g_{y} \int_{0}^{T_{s s}} e^{F_{1}(v)} f_{2}(v) d v}{-2 g_{z} \int_{0}^{T_{s w}} e^{F_{1}(v)} \frac{f_{2}(v)}{z(v)} d v+g_{z} \int_{0}^{T_{s s}} e^{F_{1}(v)} \frac{f_{2}(v)}{z(v)} d v}
\end{array}\right.
$$

were $\hat{y}_{d b}\left(T_{s s}\right)$ and $\hat{\dot{y}}_{d b}\left(T_{s s}\right)$ are obtained applying the transformation (5) respectively to $y_{d b}\left(T_{s s}\right)$ and $\dot{y}_{d b}\left(T_{s s}\right)$.

Equations (17) is used to compute $T_{s w} \leq T_{s s}$ and $\gamma$ so that the single support trajectory would "join" the double support candidate function at time $T_{s s}$. Then, the trajectory of the whole movement along the $y$ direction can be expressed as follows:

$$
y_{t r}(t)= \begin{cases}y(t) & \text { if } 0 \leq t \leq T_{s s} \\ y_{d b}(t) & \text { if } T_{s s}<t \leq T_{t r}\end{cases}
$$

Fig. 5 reports the candidate function (in green) and the single support trajectory (in blue) for the movement along the $\mathrm{y}$ direction computed for the $\mathrm{z}$ trajectory reported in Fig. 3 .

It is important to note that $x_{t r}(t), \dot{x}_{t r}(t), y_{t r}(t)$ and $\dot{y}_{t r}(t)$ are continuous in $0 \leq t \leq T_{t r}$. In fact, continuity at $t=T_{s s}$ is guaranteed by Eq. (13) and (17), while for $t \neq T_{s s}$, it is guaranteed based on the definition Eq. (3), (12) and (16). This condition ensure the absence of impulsive acceleration which may cause loss of balance. 


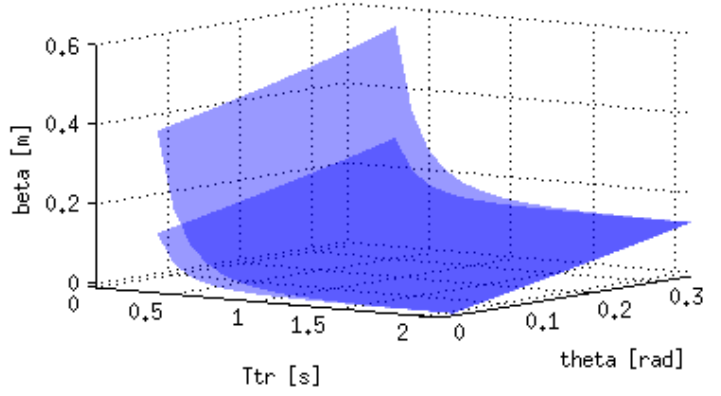

Fig. 6. $\quad \beta$ parameter for $d=0.16 m$ and $d=0.40 m$

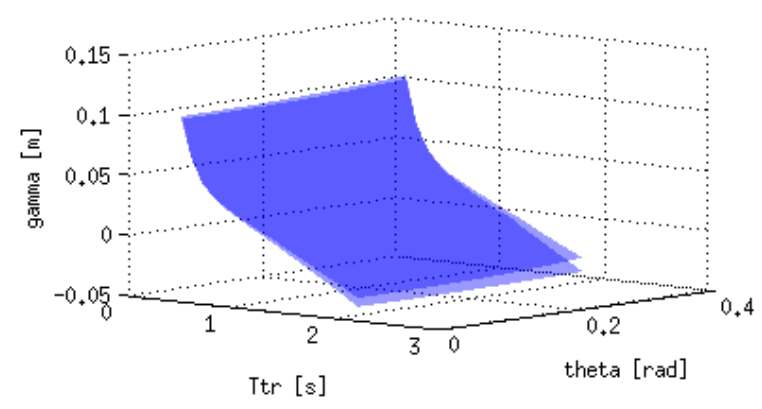

Fig. 7. $\quad \gamma$ parameter for $d=0.16 \mathrm{~m}$ and $d=0.40 \mathrm{~m}$

\section{Analysis of the Transition Movement}

Equations (15) and (18) allow one to compute the trajectory of the whole transition movement of the COM along the $\mathrm{x}$ and $\mathrm{y}$ direction. The existence of such trajectories depends on the existence of a ZMP trajectory whose parameters can be computed using (13) and (17). However, in order to preserve the balance of the real robot during the transfer movement, the ZMP must be inside the convex hull of the support regions. We therefore define the feasible range of values for the ZMP parameters according to the dimension of the foot of the COMAN robot: $-0.06 \leq \beta \leq 0.09$ and $-0.03 \leq \gamma \leq 0.03$. Fig. 6 and 7 report the value of $\beta$ and $\gamma$, computed for different gaits. The two surfaces in Fig. 6 represent the value of $\beta$ while the step length $|d|$ is fixed (see III-A) and the slope inclination and the desired duration (respectively $\theta$ and $T_{t r}$ ) change. In particular, the lower surface corresponds to the smallest step length that the COMAN robot can perform in order to have one foot in front of the other $(|d|=0.16 \mathrm{~m})$ and the surface on top of it, refers to a step length equal to $0.40 \mathrm{~m}$. We can notice that the parameter has a non linear dependence on $T_{t r}$ and $|d|$ : it increases when the desired duration decreases or the step length increases. In fact, faster motions require larger accelerations that can be generated by moving the ZMP away from the anchor point of the inverted pendulum. In Fig. 7 the dependency of parameter $\gamma$ on the gait is presented. The two surfaces represent the values of $\gamma$ for the same step lengths considered in Fig. 6. The dependency of $\gamma$ on the step length is mainly due to $T_{s s}$ which changes according to the forward movement.

Furthermore, both parameters have a dependency on the slope inclination (although this dependency is low for $\gamma$ ). In fact, by changing $\theta$, the dynamics of the movement along the vertical direction alters too. A higher inclination requires a larger vertical acceleration, which can be generated with a ZMP moving away from the anchor point of the inverted pendulum.

When the values of $\beta$ and $\gamma$ (computed for a desired gait) exceed the feasible range, different strategies can be used. As suggested by the analysis of figures 6 and 7, one solution consists of finding a feasible gait close to the desired one, for example changing the step length or the duration of the transfer movement. Another solution could be to modify the functions (12) and (16).

\section{EXPERIMENTS}

\section{A. The COMAN Robot}

The lower body of the COMAN robot was used to perform the experiments reported in this section. This is a bipedal robot which is powered by actuators implementing joints with physical compliance [9]. All the joints of the lower body are compliant with the exception of the joints located at the ankle roll, hip roll and hip yaw.

Each joint has torque sensor and three encoders to measure the position of the joint before and after the elastic transmission while the third is an absolute encoder sensor used for the system initialization. Motors are position controlled with a PID control loop [10]. Moreover the COMAN robot is equipped with a 6 axis IMU located at the pelvis and two custom 6 axis force/torque sensors mounted at the feet, below the ankles.

The disturbances can be quite important when the steps are large, which is compulsory in dynamic transition phases. For this reason, we use sensory feedback from the robot to add active compliance in order to smoothen the motion of the robot. To do so, we apply the controller described in [11], which combines the control law of [12] that emulates a compliant behavior of the center of mass using the information from the force/torque sensors in the robot feet, and a compliant attitude control algorithm based on feedback from the inertia measurement unit rigidly linked to the pelvis. This algorithm is not intended to directly increase the stability of walking motions, but it does so by acting like a low-pass filter to disturbances applied the robot, such as landing impacts which are perturbations that our model does not take into account.

\section{B. Experimental results}

The experiment reported in this section permitted the COMAN robot to walk on a flat ground for a few steps then a slope of $10^{\circ}$ inclination occurs. The trajectory of the COM (supposed rigidly connected at the pelvis of the robot) was computed at the beginning of each step, then the trajectories for the feet to respect of the pelvis of the robot was calculated and processed by the controller. Using inverse kinematics, joint references were computed and sent to the joints of the robot.

The walking gait parameters during the different phases and the parameters generated from the trajectory generator during the transition are collected in Table I. As it can be observed, ZMP parameters change considerably during the transition in order to allow the robot to perform the desired movement. In Fig. 8 the reference trajectory of the right foot 
TABLE I

GAITS USED DURING THE EXPERIMENT

\begin{tabular}{lccc}
\hline & Flat & Transition & Incline \\
\hline Step length [m] & 0.19 & 0.19 & 0.09 \\
$T_{s s}[\mathrm{~s}]$ & 0.76 & 0.78 & 0.75 \\
$T_{d b}[\mathrm{~s}]$ & 0.20 & 0.27 & 0.20 \\
$\beta[\mathrm{m}]$ & 0.005 & 0.082 & 0.0 \\
$\gamma[\mathrm{m}]$ & 0.0 & 0.011 & 0.0 \\
$T_{s w}[\mathrm{~s}]$ & 0.380 & 0.132 & 0.380 \\
\hline
\end{tabular}

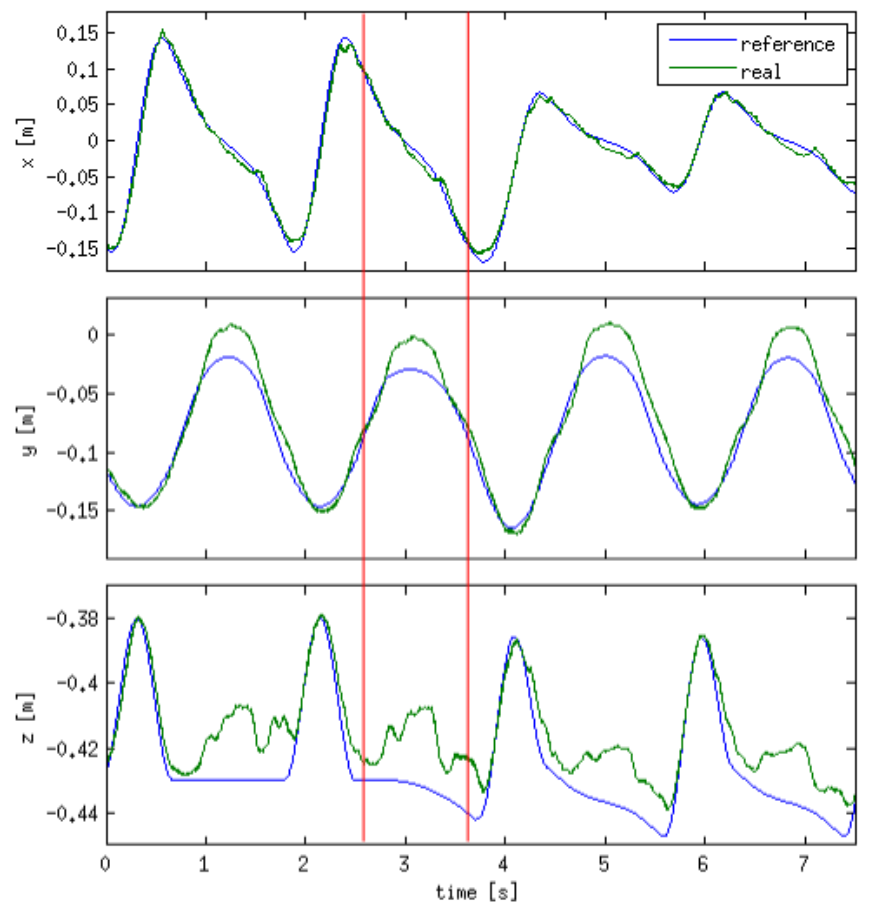

Fig. 8. Foot trajectories for the right leg

is represented in blue and the real position assumed by the right foot is reported in green. It was computed processing the angle values of the joints using the forward kinematics. The transition phase starts at $2.7 \mathrm{~s}$ and ends at $3.7 \mathrm{~s}$ as indicated by the two red lines in the graph. The passive compliance and the control action contribute to increase the robustness of the walking adapting the robot to the uncertainties of the ground. In fact, the real trajectory differs from the desired one, especially along the $\mathrm{y}$ and $\mathrm{z}$ direction. The trajectory developed by the trajectory generator allows the robot to perform the transition movements without fast acceleration or deceleration that may generate instability during the walking (see III-C). The movements of the robot during the experiments appears smooth and the change of the walking plane did not affect the walking periodicity. It is evident also by looking the foot position profile in Fig. 8 .

\section{CONCLUSIONS AND FUTURE WORKS}

We addressed the problem of dynamic walking on inclined planes. In this paper we focused on the case where the inclination changes just in the walking direction. The problem is divided into two phases: walking and transition. The first is solved using traditional linear models for walking trajectory generation. The transition phase requires a deeper study because of the intrinsic complexity of the motion that must be performed. The dynamics of the robot was approximated by a (non linear) inverted pendulum. Then, the equations of this model were defined and an "equivalent" model was used to develop a trajectory generator. Using this trajectory generator we enabled the COMAN robot to perform a dynamic transition from a horizontal surface to a slope of $10^{\circ}$. The motion generated achieves a smooth and fast transition between the two surfaces, proving that our model captures well the dynamics of the real system. The passive compliance improved the robustness of the walking adapting the robot motion to the uncertainties of the ground. The novel method generates transition between planes generally oriented, hence, it can be used to perform a dynamic transition from horizontal surface to a downslope surface and from a slope surface to another surface with different inclination as well. Based on these results, our goal is to extend our work and enable the robot to perform locomotion on challenging uneven terrains by planning appropriate trajectories and executing them in a controlled and adaptable way.

\section{REFERENCES}

[1] S. Kajita, F. Kanehiro, K. Kaneko, K. Fujiwara, K. Harada, K. Yokoi, and H. Hirukawa, "Biped walking pattern generation by using preview control of zero-moment point," in Robotics and Automation, 2003. Proceedings. ICRA '03. IEEE International Conference on, vol. 2, Sept., pp. $1620-1626$ vol.2.

[2] S. Kajita, F. Kanehiro, K. Kaneko, K. Yokoi, and H. Hirukawa, "The $3 \mathrm{~d}$ linear inverted pendulum mode: a simple modeling for a biped walking pattern generation," in Intelligent Robots and Systems, 2001. Proceedings. 2001 IEEE/RSJ International Conference on, vol. 1, pp. 239-246 vol.1.

[3] C. Lutz, F. Atmanspacher, A. Hornung, and M. Bennewitz, "Nao walking down a ramp autonomously," in Intelligent Robots and Systems (IROS), 2012 IEEE/RSJ International Conference on, Oct., pp. 51695170.

[4] B. Lim, M. Lee, J. Kim, J. Lee, J. Park, K. Seo, and K. Roh, "Control design to achieve dynamic walking on a bipedal robot with compliance," in Robotics and Automation (ICRA), 2012 IEEE International Conference on, May, pp. 79-84.

[5] U. Seven, T. Akbas, K. Fidan, M. Yilmaz, and K. Erbatur, "Humanoid robot walking control on inclined planes," in Mechatronics (ICM), 2011 IEEE International Conference on, April, pp. 875-880.

[6] L. Colasanto, N. Tsagarakis, and D. Caldwell, "A compact model for the compliant humanoid robot coman," in Biomedical Robotics and Biomechatronics (BioRob), 2012 4th IEEE RAS EMBS International Conference on, June 2012, pp. 688-694.

[7] L. Colasanto, N. Tsagarakis, Z. Li, and D. Caldwell, "Internal model control for improving the gait tracking of a compliant humanoid robot," in Intelligent Robots and Systems (IROS), 2012 IEEE/RSJ International Conference on, Oct., pp. 5347-5352.

[8] E. Spyrakos-Papastavridis, G. A. Medrano-Cerda, N. G. Tsagarakis, J. S. Dai, and D. G. Caldwell, "A compliant humanoid walking strategy based on the switching of state feedback gravity compensation controllers," in Intelligent Robots and Systems (IROS), 2013 IEEE/RSJ International Conference on, 2013, pp. 3630-3636.

[9] N. Tsagarakis, Z. Li, J. Saglia, and D. Caldwell, "The design of the lower body of the compliant humanoid robot "ccub"," in Robotics and Automation (ICRA), 2011 IEEE International Conference on, May, pp. 2035-2040

[10] N. Tsagarakis, F. Becchi, L. Righetti, A. Ijspeert, and D. Caldwell, "Lower body realization of the baby humanoid - icub," in Intelligent Robots and Systems, 2007. IROS 2007. IEEE/RSJ International Conference on, Oct 2007, pp. 3616-3622.

[11] N. Perrin, N. Tsagarakis, and D. G. Caldwell, "Compliant attitude control and stepping strategy for balance recovery with the humanoid coman," in Intelligent Robots and Systems, 2013. IROS 2013. IEEE/RSJ International Conference on, 2013.

[12] Z. Li, B. Vanderborght, N. Tsagarakis, L. Colasanto, and D. Caldwell, "Stabilization for the compliant humanoid robot coman exploiting intrinsic and controlled compliance," in Robotics and Automation (ICRA), 2012 IEEE International Conference on, May, pp. 2000-2006. 